\title{
Metastatic Sternal Osteosarcoma: A Rare Tumor
}

\author{
Muhammad Masab ${ }^{1}$, Ena Arora ${ }^{2}$, Sorab Gupta ${ }^{3}$, Hafsa Farooq ${ }^{4}$, Vishal Jindal ${ }^{5}$, Shorabh Sharma 6 \\ 1. Internal Medicine, Albert Einstein medical center 2. Internal Medicine, Government Medical College and Hospital \\ Chandigarh 3. Hematology/oncology, Albert Einstein Medical Center 4. Internal Medicine, Waterbury Hospital 5. \\ Internal Medicine, St. Vincent Hospital Worcester 6. Internal Medicine, SBH Health System
}

Corresponding author: Muhammad Masab, masabmuh@einstein.edu

\begin{abstract}
Osteosarcoma is the most common primary malignant tumor of the long bones. However, primary osteosarcoma of the chest wall, particularly the sternum, is an extremely rare occurrence. We report a 36year-old male presenting with a hard, immobile, palpable, anterior chest wall mass. A computed tomographic (CT) scan showed a large destructive anterior mediastinal mass involving the manubrium and sternum with multiple bilateral calcified lung masses, pleural effusions and partially calcified aortopulmonary, right hilar and subcarinal lymphadenopathy. Incisional biopsy of the mass revealed grade 2 chondroblastic osteosarcoma. The patient underwent one cycle of chemotherapy with ifosfamide and palliative radiation. Unfortunately, the patient was unable to tolerate ifosfamide and developed severe nausea and vomiting requiring the discontinuation of chemotherapy. Given his metastatic disease and inability to tolerate standard chemotherapy, he was referred to a comprehensive cancer center for advanced clinical trials.
\end{abstract}

Categories: Internal Medicine, Oncology, Pulmonology

Keywords: osteosarcoma, chest wall tumor, sternal tumor, pulmonary metastasis

\section{Introduction}

Primary osteosarcoma of the sternum is an extremely rare tumor, with a reported median age of 42 years at the time of diagnosis [1]. It is considered an aggressive malignant tumor with poor prognosis [2]. We present a unique case of primary osteosarcoma of the sternum with metastasis to the lungs. Osteosarcoma should always be considered in the differential diagnosis of an anterior chest wall mass. Given the aggressive nature of this tumor and the poor prognosis associated with metastatic disease, early diagnosis and management are of paramount importance.

Received 02/08/2018 Review began 02/13/2018 Review ended 02/14/2018 Published 02/19/2018

๑) Copyright 2018 Masab et al. This is an open access article distributed under the terms of the Creative Commons Attribution License CC-BY 3.0., which permits unrestricted use, distribution, and reproduction in any medium, provided the original author and source are credited.

\section{Case Presentation}

A 36-year-old African American male with no significant past medical history presented to our cancer center with a six-month history of an anterior chest wall mass. His other symptoms included chest pain, cough, and an unintentional weight loss of $5 \mathrm{lbs}$ over a period of one month. The physical exam was significant for a hard, palpable mass on the anterior chest overlying the sternum, fixed to the chest wall and extending $20 \mathrm{~cm}$ horizontally and $14 \mathrm{~cm}$ vertically. The laboratory analysis was significant for mild anemia with $\mathrm{Hb} 12.7 \mathrm{~g} / \mathrm{dl}$ (13.5-17.5 g/dl), elevated lactate dehydrogenase (LDH) with a value of 908 U/L (140-260 $\mathrm{U} / \mathrm{L})$, and elevated alkaline phosphatase (ALP) with a value of $418 \mathrm{IU} / \mathrm{L}$ (44-147 IU/L). A computed tomography (CT) scan of the chest and abdomen with contrast revealed a large $(11.5 \times 15.9 \times 11.9 \mathrm{~cm})$ destructive anterior mediastinal mass involving the manubrium and sternum, extending anteriorly into the pectoral muscles (Figure 1). 


\section{Cureus}

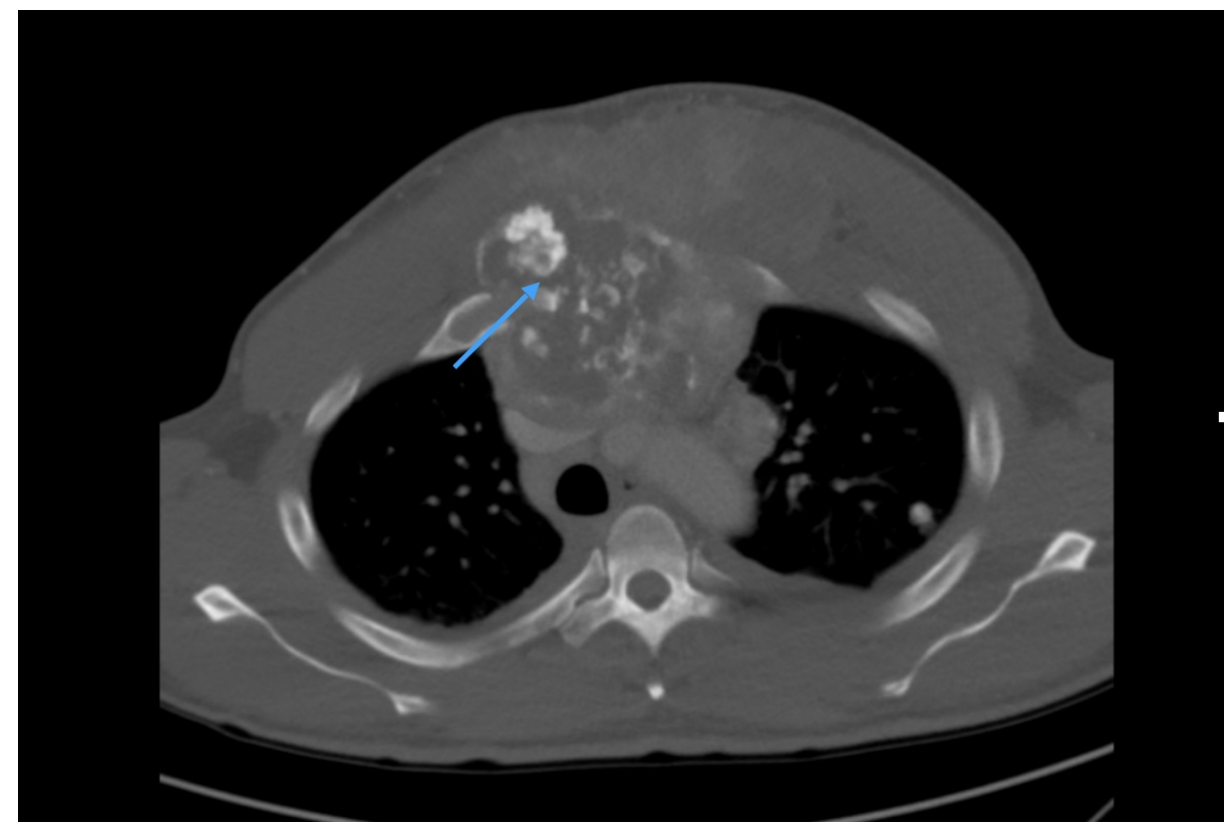

FIGURE 1: Computed tomography (CT) of the chest with contrast revealing a $11.5 \times 15.9 \times 11.9 \mathrm{~cm}$ anterior calcified mediastinal mass (blue arrow) involving the manubrium and sternum, extending anteriorly into the pectoral muscles

It also showed multiple bilateral calcified lung masses, the largest one located in the left lower lobe measuring 6.3 x $5.1 \mathrm{~cm}$ (Figure 2).

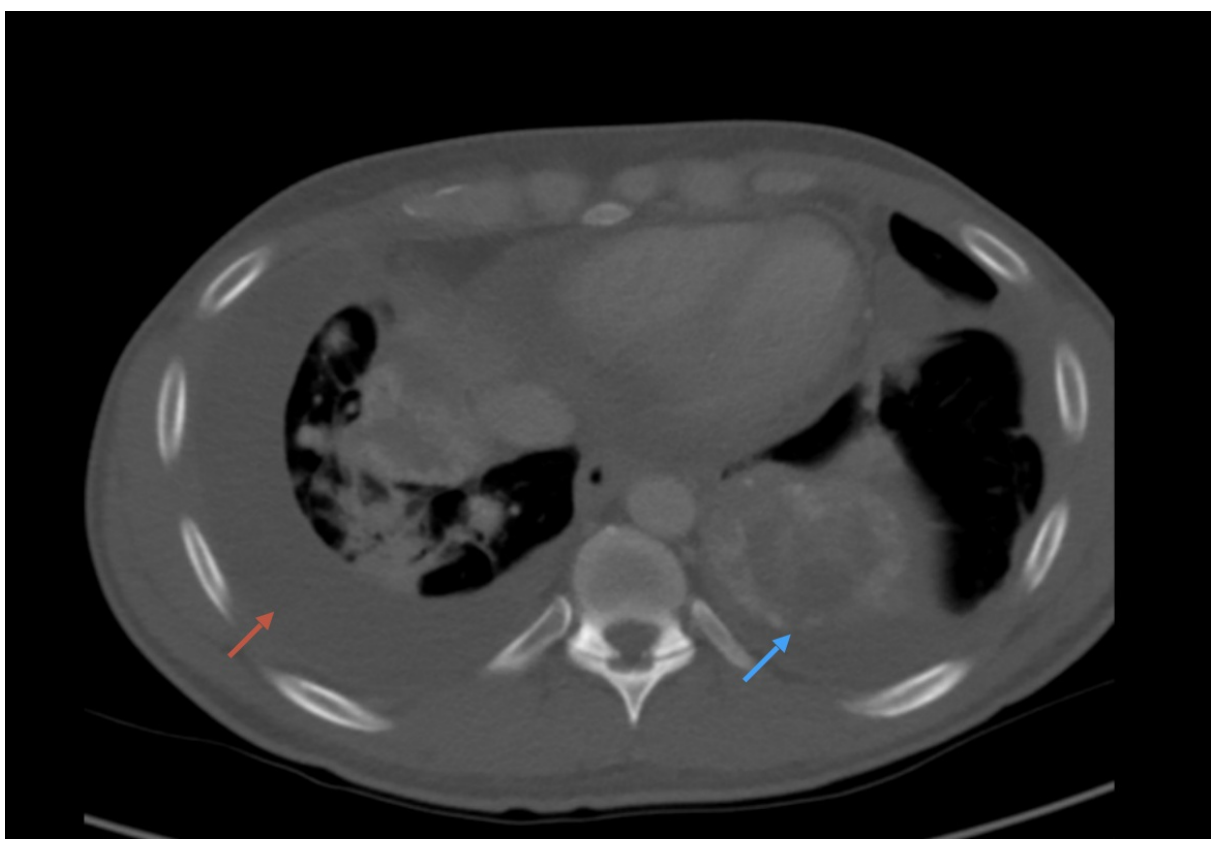

FIGURE 2: Computed tomography (CT) of the chest with contrast illustrating the bilateral pulmonary metastatic lesions with a large left lower lobe lung mass (blue arrow), bilateral pleural (red arrow) and pericardial effusions

There were bilateral pleural effusions, pericardial effusion and partially calcified aortopulmonary, right hilar and subcarinal lymphadenopathy. He underwent diagnostic thoracentesis which was negative for malignant 
cells, and incisional biopsy of the mediastinal mass that came back positive for grade 2 chondroblastic osteosarcoma. He also underwent biopsy of the rapidly growing lung lesion which was consistent for chondroblastic osteosarcoma. He was started on first-line chemotherapy with single-agent ifosfamide and underwent a brief course of palliative radiation therapy. Unfortunately, the patient was unable to tolerate ifosfamide and developed severe nausea and vomiting requiring the discontinuation of chemotherapy. He was planned to start on cisplatin and adriamycin but refused further treatment as he was worried about serious side effects. Given his metastatic disease and inability to tolerate standard chemotherapy, he was referred to a comprehensive cancer center for advanced clinical trials.

\section{Discussion}

Chest wall tumors are classified into four main groups: primary, metastatic, extension from adjacent structures or non-neoplastic lesions. Primary chest wall osteosarcoma is a rare entity and constitutes $10 \%$ of all primary chest wall tumors. It accounts for almost $3 \%$ of all sarcomas. It is considered an aggressive malignant tumor with poor prognosis. The occurrence of osteosarcoma in the sternum is extremely rare, with a reported median age of 42 years at the time of diagnosis [1].

Patients with osteosarcoma of the sternum typically present with a rapidly enlarging painful mass, with or without a history of radiation exposure. In many cases, they may also be completely asymptomatic. These tumors do have a metastatic potential and it is reported that $34 \%$ of patients have metastasis at the time of presentation [1]. Osteosarcomas present both as locally advanced disease as well as with distant metastasis. The initial workup includes imaging of the primary site (plain radiographs, CT and/or magnetic resonance imaging (MRI)), and additional imaging for staging purpose including positron emission tomography (PET), CT, and bone scan.

Plain radiographs of osteosarcoma show irregular reactive bone and cortical destruction. A bone scan may show additional synchronous lesions. An MRI provides excellent soft-tissue contrast and may be essential for operative planning. It is the best imaging modality to define the extent of the lesion within the bone, as well as the extra osseous spread of tumor including soft tissue extension and "skip" metastases. In addition, ALP and LDH levels are frequently elevated in patients with osteosarcoma, especially with metastatic disease [3].

Management depends on the extent of the disease. Preoperative chemotherapy followed by wide excision of the primary tumor is recommended for localized disease. Chemotherapy and metastasectomy (pulmonary, visceral or skeletal) are included as options for the management of metastatic disease. An unresectable metastatic disease requires management with chemotherapy and/or radiation therapy.

Although chemotherapy is associated with improved outcomes in patients with localized osteosarcoma, the results are significantly poorer in patients with metastatic disease at presentation, resulting in a very poor prognosis [4]. Bacci et al. studied 44 patients with metastatic osteosarcoma (with pulmonary metastasis) who received neoadjuvant chemotherapy followed by surgery and adjuvant chemotherapy. The five-year overall survival and disease-free survival rates for all 44 patients were $14 \%$ and $17 \%$ respectively. These were much worse than those achieved with the same chemotherapy protocol in 144 patients with localized disease at presentation (79\% overall survival and $73 \%$ disease-free survival) [5].

In a study of 57 patients with metastatic disease at presentation treated with cisplatin, doxorubicin, and high dose of methotrexate and ifosfamide, the two-year event-free survival and overall survival rates were $21 \%$ and $55 \%$, respectively, compared to $75 \%$ and $94 \%$ in patients with non-metastatic disease at presentation, treated with the same chemotherapy protocol [6]. Among patients with primary metastases treated in cooperative osteosarcoma trials, long-term survival rates were higher for patients whose metastases were excised following chemotherapy and surgery of the primary tumor compared to those patients with unresectable metastases ( $48 \%$ and $5 \%$ respectively).

\section{Conclusions}

This case is presented to highlight the diagnosis and management of chest wall osteosarcoma. Primary osteosarcoma of the sternum is a rare tumor. The physicians should always consider the possibility of a malignancy in patients presenting with an anterior chest wall mass. Given the aggressive nature of the tumor and poor prognosis, early recognition and prompt treatment are critical.

\section{Additional Information \\ Disclosures}

Human subjects: All authors have confirmed that this study did not involve human participants or tissue. Conflicts of interest: In compliance with the ICMJE uniform disclosure form, all authors declare the following: Payment/services info: All authors have declared that no financial support was received from any organization for the submitted work. Financial relationships: All authors have declared that they have no financial relationships at present or within the previous three years with any organizations that might have an interest in the submitted work. Other relationships: All authors have declared that there are no 


\section{Cureus}

other relationships or activities that could appear to have influenced the submitted work.

\section{References}

1. Rad MP, Masoum SH, Layegh P, Rad MS: Primary osteosarcoma of the sternum: a case report and review of the literature. Arch Bone Jt Surg. 2014, 2:272.

2. Moghazy K, Al-Jehani Y, El-Baz A, El-Ghoneimy Y: Incidental finding of a large chest wall osteosarcoma-a case report. Gulf J Oncolog. 2007, 1:93-7.

3. Bacci G, Longhi A, Ferrari S, et al.: Prognostic significance of serum lactate dehydrogenase in osteosarcoma of the extremity: experience at Rizzoli on 1421 patients treated over the last 30 years. Tumori. 2004, 90:47884.

4. Meyers PA, Heller G, Healey JH, et al.: Osteogenic sarcoma with clinically detectable metastasis at initial presentation. J Clin Oncol. 1993, 11:449-453. 10.1200/JCO.1993.11.3.449

5. Bacci G, Briccoli A, Mercuri M, et al.: Osteosarcoma of the extremities with synchronous lung metastases: long-term results in 44 patients treated with neoadjuvant chemotherapy. J Chemother. 1998, 10:69-76. 10.1179/joc.1998.10.1.69

6. Bacci G, Briccoli A, Rocca M, et al.: Neoadjuvant chemotherapy for osteosarcoma of the extremities with metastases at presentation: recent experience at the Rizzoli Institute in 57 patients treated with cisplatin, doxorubicin, and a high dose of methotrexate and ifosfamide. Ann Oncol. 2003, 14:1126-1134.

10.1093/annonc/mdg286 